\title{
Slow modulation of ongoing discharge in the auditory cortex during an interval-discrimination task
}

\author{
Juan M. Abolafia ${ }^{1}$, Marina Martinez-Garcia ${ }^{2}$, Gustavo Deco ${ }^{2,3}$ and Maria V. Sanchez-Vives ${ }^{1,3}$ * \\ 1 Institut d'Investigacions Biomèdiques August Pi i Sunyer, Barcelona, Spain \\ ${ }^{2}$ Computational Neuroscience Group, Department of Information and Communication Technologies, Universitat Pompeu Fabra, Barcelona, Spain \\ ${ }^{3}$ Institució Catalana de Recerca i Estudis Avançats, Barcelona, Spain
}

Edited by:

Agnes Gruart, University Pablo de Olavide, Spain

\section{Reviewed by:}

Israel Nelken, Hebrew University, Israel

Manuel S. Malmierca, University of Salamanca, Spain

\section{*Correspondence:}

Maria V. Sanchez-Vives, Institut d'Investigacions Biomèdiques August Pi i Sunyer, Roselló 149-153, 08036 Barcelona, Spain.

e-mail: msanche3@clinic.ub.es; sanchez.vives@gmail.com
In this study, we recorded single unit activity from rat auditory cortex while the animals performed an interval-discrimination task. The animals had to decide whether two auditory stimuli were separated by either 150 or $300 \mathrm{~ms}$, and go to the left or right nose poke accordingly. Spontaneous firing in between auditory responses was compared in the attentive versus non-attentive brain states. We describe the firing rate modulation detected during intervals while there was no auditory stimulation. Nearly $18 \%$ of neurons $(n=14)$ showed a prominent neuronal discharge during the interstimulus interval, in the form of an upward or downward ramp towards the second auditory stimulus. These patterns of spontaneous activity were often modulated in the attentive versus passive trials. Modulation of the spontaneous firing rate during the task was observed not only between auditory stimuli, but also in the interval preceding the stimulus. These slow modulatory components could be locally generated or the result of a top-down influence originated in higher associative association areas. Such a neuronal discharge may be related to the computation of the interval time and contribute to the perception of the auditory stimulus.

Keywords: auditory, decision-making, spontaneous, awake, rat

\section{INTRODUCTION}

Sensory areas such as primary auditory cortex are primarily associated with stimulus encoding and there are different aspects of neuronal responses relevant to this function. Spike count (Polley et al., 2004; Weinberger, 2004; Fritz et al., 2005, 2007; Nelken et al., 2005), spike timing (Kayser et al., 2010), a combination of both spike count and spike timing (Lu and Wang, 2004; Imaizumi et al., 2010), or neuronal firing pattern (Kayser et al., 2009) have all been associated to cortical auditory encoding. However there is increasing evidence that early cortices, and in particular auditory cortex, are not only feature detectors. Multimodal responses (Kayser et al., 2008; Lemus et al., 2010), attentional modulation (Hubel et al., 1959; Gottlieb et al., 1989; Otazu et al., 2009), expectation (Jaramillo and Zador, 2011), or reward-modulation (Shuler and Bear, 2006) illustrate additional contextual aspects that modulate responses even in early sensory cortices. While fast responses to auditory stimuli have been characterized in detail in auditory cortex, the slow modulation of neuronal firing to evoked and spontaneous activity has barely been studied. Slow modulation of sustained responses has been found to predict the behavioral decisions during auditory categorization tasks in monkeys, including errors (Selezneva et al., 2006). Sensory or behavioral events contingent on reinforcement can also result on slow modulation of firing rate or sustained firing as a consequence of a learning process (Brosch et al., 2011b). Therefore, slow modulation of firing could constitute an anticipatory mechanism that associates events (stimulus-behavior-reinforcer) that are relevant or adaptive to the environment. These cognitive components associated to stimulus discrimination tasks have been more commonly associated to higher areas such as frontal areas (Romanski and Goldman-Rakic, 2002; Lemus et al., 2009).

In the present study we recorded the activity of 86 neurons from the auditory cortex of the behaving rat. We aimed at exploring the slow modulation of neuronal firing in the intervals between stimuli while the rat was performing an interval-based decision-making task.

\section{MATERIALS AND METHODS}

Single unit recordings from two Lister Hooded rats were obtained by means of chronically implanted tetrodes in the primary auditory cortex (Doron et al., 2002). Surgical protocol and recordings were the same as the ones described in (Abolafia et al., 2011). Rats were cared for and treated in accordance with the Spanish regulatory laws (BOE 256; 25 October 1990) which comply with the EU guidelines on protection of vertebrates used for experimentation (Strasbourg 18 March 1986).

\section{EXPERIMENTAL SET UP}

The recordings were performed inside a box built in black acrylic with a surface of $22 \mathrm{~cm}(\mathrm{~L}) \times 25.5 \mathrm{~cm}(\mathrm{~W}) \times 35 \mathrm{~cm}(\mathrm{H})$. The box in which the recordings were performed was placed inside two wooden boxes placed inside the other. Between each box, two isolating foam rubbers ( 4 and $2 \mathrm{~cm}$ thick) were placed to soundproof for low and high frequencies. A wooden lid and equal soundproof foams closed the whole recording chamber. A hole permitted the entry of a recording wire ( $2 \mathrm{~mm}$ thick) connected to a preamplifier. Water-valves were placed outside the wooden boxes. Animals poked their nose into three different sockets, each one $2 \mathrm{~cm}$ wide 
and separated by $3 \mathrm{~cm}$. The top part of the socket did not have a lid to avoid being hit with the microdrive. During the recordings, the rat could move freely within the limited space of the chamber. Recordings were obtained in the dark, and the experiment was filmed with an infrared camera placed above the recording chamber.

\section{BEHAVIORAL PROTOCOL}

The experimental procedure consisted of a sequence of different recording stages, including passive and attentive stages and with a total duration of $c a .3 \mathrm{~h}$. Animals only went through the whole session once a day.

A tuning curve (ca. $24 \mathrm{~min}$ ) and a passive listening recording stage $(c a .17 \mathrm{~min})$ were performed before and after the attentive stage (ca. $40 \mathrm{~min}$ ). The final stage comprised a passive recording with a reward ( $c a .40 \mathrm{~min}$ ) delivery after each pair of stimuli was presented. The aim was to compare the neuronal responses while the idle animal listened to stimuli presentation with respect to the attentive brain state during task performance. In the attentive task, the animal was trained to poke its nose into the center socket which immediately triggered the onset of two identical stimuli ( $80 \mathrm{~dB}, 5.3 \mathrm{kHz}, 50 \mathrm{~ms}$ duration). The animal had to remain in the center socket until the end of the stimuli presentation. The animals had to discriminate whether the two stimuli were separated (from the end of stimulus 1 to beginning of stimulus 2) by 150 or $300 \mathrm{~ms}$. This required a left or a right nose poke in order to get a water reward. In the attentive task, false alarms (poking in the opposite side) or early withdrawals (withdrawal before stimuli termination) were punished with a 3 -s timeout and a white noise (WAV-file, $0.5 \mathrm{~s}, 80 \mathrm{~dB}$ SPL). Passive stages had the same amount of trials (180 trials each side), stimuli $(50 \mathrm{~ms} ; 80 \mathrm{~dB} ; 5.3 \mathrm{kHz})$, interstimulus interval (150 and $300 \mathrm{~ms}$ ), and intertrial interval (2-3 s) as for the attentive task.

Animals were implanted and recorded whenever they reached $75 \%$ correct trials.

\section{PRESENTATION OF SOUND STIMULI}

Protocols of stimulation were controlled through Matlab ${ }^{\circledR}$, a National Instrument card (BNC-2110), and a breakout box (FS $300 \mathrm{kHz}$ ). Sound triggers had microseconds precision. Sound stimuli were delivered through earphones (ER.6i Isolator, Etymotic Research Inc.) screwed in each recording session to earphone holders chronically attached to the animal skull with dental cement. The earphones were adjusted inside the ear with a silicone tip which allowed the isolation from any sound unrelated to the protocol. Sound calibration was performed with a microphone (MM1, Beyerdynamic) placed $1 \mathrm{~mm}$ away from the earphone, and a preamplifier (USB Dual Pre, Applied Research and Technology). The sound stimuli during the passive and attentive recording stages had a 50-ms duration, an intensity of $80 \mathrm{dBs}$ SPL, and pure tones of $5322 \mathrm{~Hz}$ with a 6-ms rise/fall cosine ramps. It was therefore identical for both the first and second stimulus. Interstimulus intervals were 150 or $300 \mathrm{~ms}$, and both had the same amount of trials (180). Similarly, the total number of correct trials in the attentive stage was the same as in the passive one (180). The intertrial interval also had a similar duration in the attentive and passive stages $(2-3 \mathrm{~s})$.

\section{DATA ANALYSIS}

Cluster cutting and peri-stimulus time histograms (PSTH) were performed according to the methods described in (Abolafia et al., 2011). Cluster cutting (isolating single units from the multiunit recorded data) was performed using an Off-Line Spike Sorter (OFSS, Plexon). Waveforms were first sorted into units by using the valley-seeking algorithm (Koontz and Fukunaga, 1972). Waveforms were considered to have been generated by a single neuron when they occurred simultaneously in the four electrodes that defined a discrete cluster in 3D principal component (or peak-topeak) space distinct from clusters for other units using a MANOVA test $(p<0.05)$. Single units exhibited a recognizable refractory period $(>1 \mathrm{~ms})$ in their InterSpikeInterval histograms and had a characteristic and distinct waveform shape and peak-to-peak amplitude when compared to other spikes. Additional criteria were used in order to isolate single units such as the difference between InterSpikeInterval histograms or the crosscorrelograms among the recorded neurons.

\section{RESULTS}

Eighty-six single units from the rat auditory cortex were isolated and classified (Recanzone, 2000; Hromadka et al., 2008) according to their phasic auditory responses as: onset $(26 \%)$, onset + offset (13\%), offset (2\%), non-responsive $(43 \%)$, suppressive $(13 \%)$, and "other" $(3 \%)$. The percentage of non-responsive neurons was similar to the one reported by means of cell-attached recordings in the head-fixed awake animal (Hromadka et al., 2008).

We designed an interval-discrimination task where the rat had to go to the left or to the right depending on the duration of the interval between stimuli (150 or 300 ms; Figure 1). The interval between stimuli was thus behaviorally relevant in this task. In this study we describe different patterns of neuronal discharges

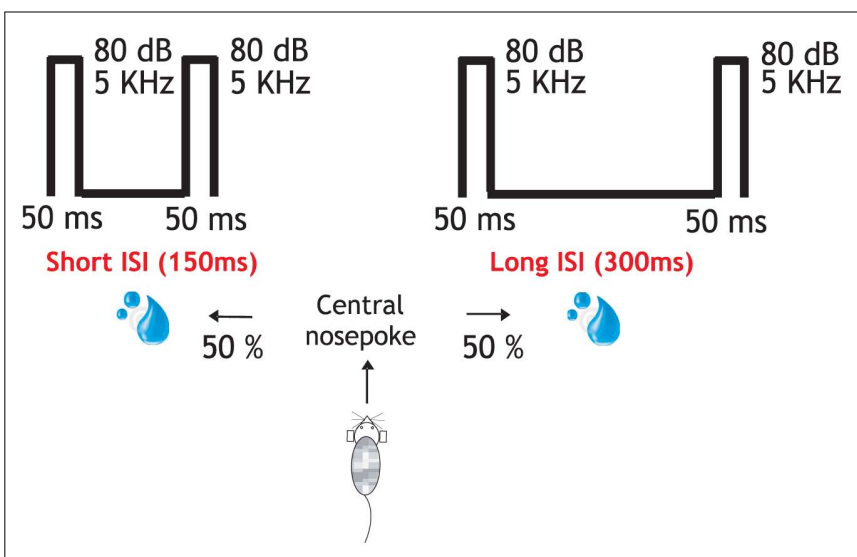

FIGURE 1 | Behavioral protocol and performance. In the attentive task the rat entered in the central socket and two identical stimuli $(50 \mathrm{~ms} ; 80 \mathrm{~dB}$; $5322 \mathrm{~Hz}$ ) were presented through earphones. About 150 or $300 \mathrm{~ms}$ interstimulus interval indicated left or right reward delivery, respectively. These same stimuli (interstimulus interval (150 and $300 \mathrm{~ms}$ ), intertrial interval (2-3s), and trial repetitions (180) were delivered under different stages: "Initial passive," attentive, "passive post" identical to the "initial passive" one and another passive recording but now followed by a reward after each pair of stimuli presentation. The total duration of the recording protocol was $3 \mathrm{~h}$. 
occurring during the intervals in between stimuli and thus in the absence of auditory stimulation. We show that the neuronal firing occurring between auditory stimuli in the auditory cortex can be quite prominent and that is often modulated by attention. Finally, the possible functional role of this slow modulation of neuronal discharge is discussed.

We found that $17.4 \%(n=14)$ of the recorded neurons showed a prominent neuronal discharge during the interstimulus interval, in the form of either an upward or downward ramp towards the second auditory stimulus. While in some of the neurons the activity during the interval ramped up toward the second stimuli ( $n=6$; Figures $\mathbf{2 A}, \mathbf{B}$, and $\mathbf{3 A}, \mathbf{B}, \mathbf{C}$ ), in others the activity ramped down following a sort of post-discharge $(n=6$; Figures $3 \mathrm{D}, \mathrm{E}$ and $4 A, B$ ). In the two remaining neurons the activity during the interval remained rather in a plateau (Figure $3 \mathrm{~F}$ ). The neurons shown here further illustrate the large heterogeneity of neuronal responses that have been described in auditory cortex.

Figure 2 illustrates the PSTHs from two different neurons while the rat was performing the task. During the passive sound stimulation, the neuron in Figure 2A had a weak offset response to the first auditory stimulus and a subsequent decrease in the firing during the interval, that progressively increased towards the second stimulus. During attention these responses became more prominent (Figure 2A; top PSTHs). The offset response was increased, and neuronal activity ramped up toward the second stimulus well above the spontaneous activity preceding the first stimulus. Interestingly, the response to the second stimulus was not an offset response but a sustained one. This is the case for both the short and the long interval trials, which were randomly given. The neuron illustrated in Figure 2B is of a different type, a "suppressive" response (Hromadka et al., 2008), since its discharge was silenced by auditory stimulation. This is well appreciated in the raster plots that correspond to the attentional trials (Figure 2B, top PSTHs). Still, even when the neuron was silenced by the auditory stimulation, its activity ramped up toward the second stimulus, more prominently in the attentional trials than in the passive ones. The second auditory stimulus again decreased its firing rate, which remained decreased for $200 \mathrm{~ms}$ following stimulation.

Out of the six neurons with increasing activity toward the second stimulus, all of them had an up-regulation of this activity during attentive trials. When the average firing rate during the first half of the interval was compared against that during the second, the activity increased in a $17 \%$ in passive trials and $246 \%$ in attentive ones for the short $(150 \mathrm{~ms})$ intervals. For the long (300 ms) intervals these values were 58 and 192\% respectively.

In some cases, the activity occurring in between auditory stimuli was not ramping-up toward the second stimulus, as the one illustrated above, but rather appeared as a prominent postdischarge following the auditory stimulation (Figures 3D,E and 4A.) In the neuron illustrated in Figure 4A, each auditory response was followed by a post-discharge lasting around $200 \mathrm{~ms}$. In this neuron, not only the auditory responses but also the auditory post-discharge was significantly increased by attention. A total of five neurons showed a similar modulation by attention, the post-discharge increasing an average of $45 \%$ (short ISIs) and 53\% (long ISIs) in attentive versus passive trials. In one neuron, the post-discharge was decreased in a $40 \%$ by attention. In the case
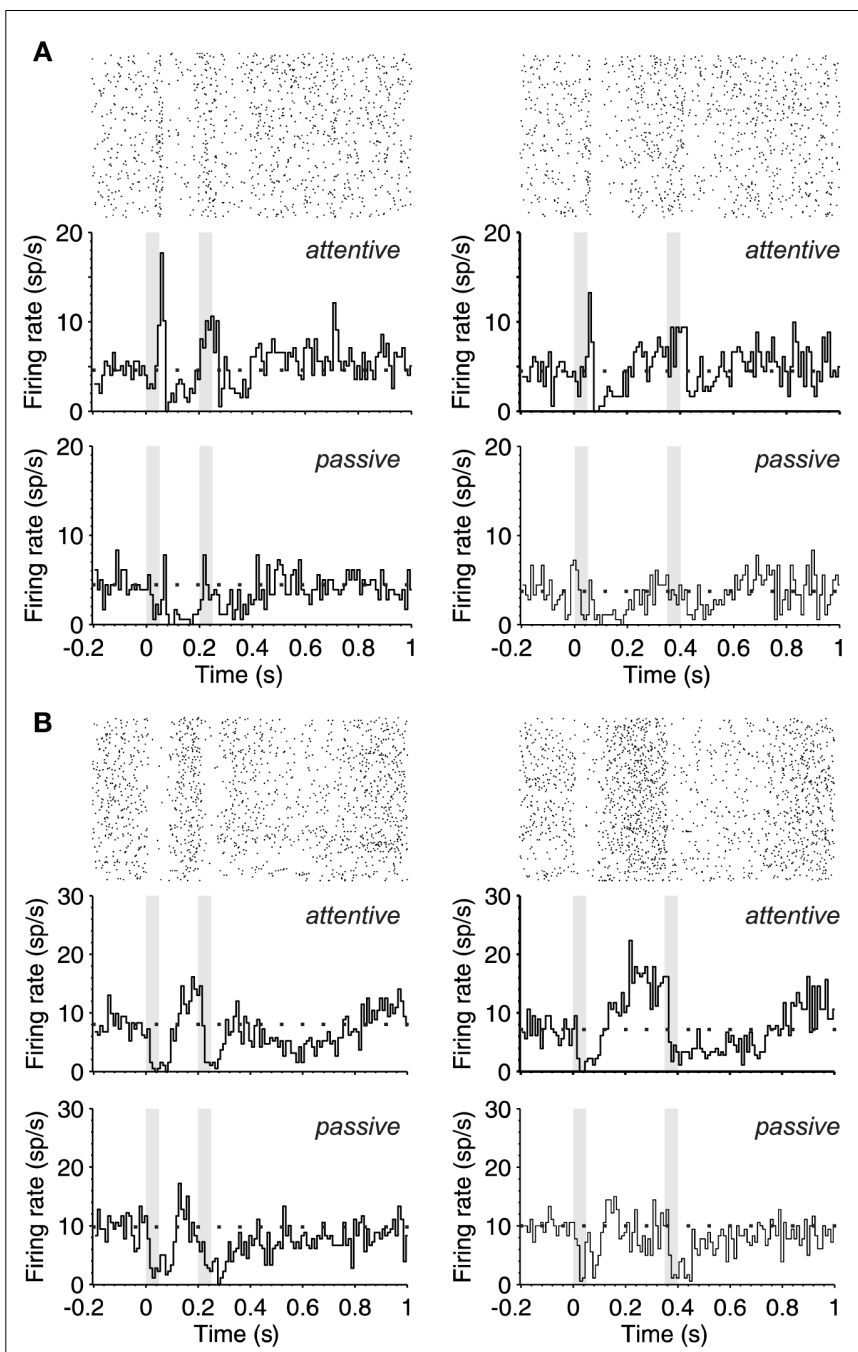

FIGURE 2 | Ramping-up activity during the interstimulus interval. (A) Upper part. PSTH (180 trials) of the response (bottom) and raster plot (top) of a single neuron during the attentive task shows the response pattern to two identical stimuli $(50 \mathrm{~ms} ; 80 \mathrm{~dB} ; 5322 \mathrm{~Hz})$ separated by $150 \mathrm{~ms}$ (left) and $300 \mathrm{~ms}$ (right). The gray boxes correspond to the periods of auditory stimulation. The PSTH shows an offset response to stimulus 1 and onset response during stimulus 2 while in the passive brain state (lower part) there is an overall decrease of excitability and no monotonic increase of spontaneous activity toward S2 presentation. (B) Same as in (A), but this other neuron is silenced by the auditory stimulation followed by an increase of firing until S2 occurrence (upper part), while in the passive brain state (lower part) the neuronal firing is markedly reduced. In $(\mathbf{A}, \mathbf{B})$ the mean spontaneous activity is represented with a dotted line.

of the neuron illustrated in Figure 4A, the firing rate during the 200-ms preceding the first auditory stimulus was also significantly increased by attention. This is the period of time that takes place when the animal is heading to the central nose poke that triggers stimulus presentation.

The firing rate during the period preceding auditory stimulation was also significantly increased during attentive trials in the neuron displayed in Figure 4B, which on the other hand had a rather different auditory response. This neuron had a weak 


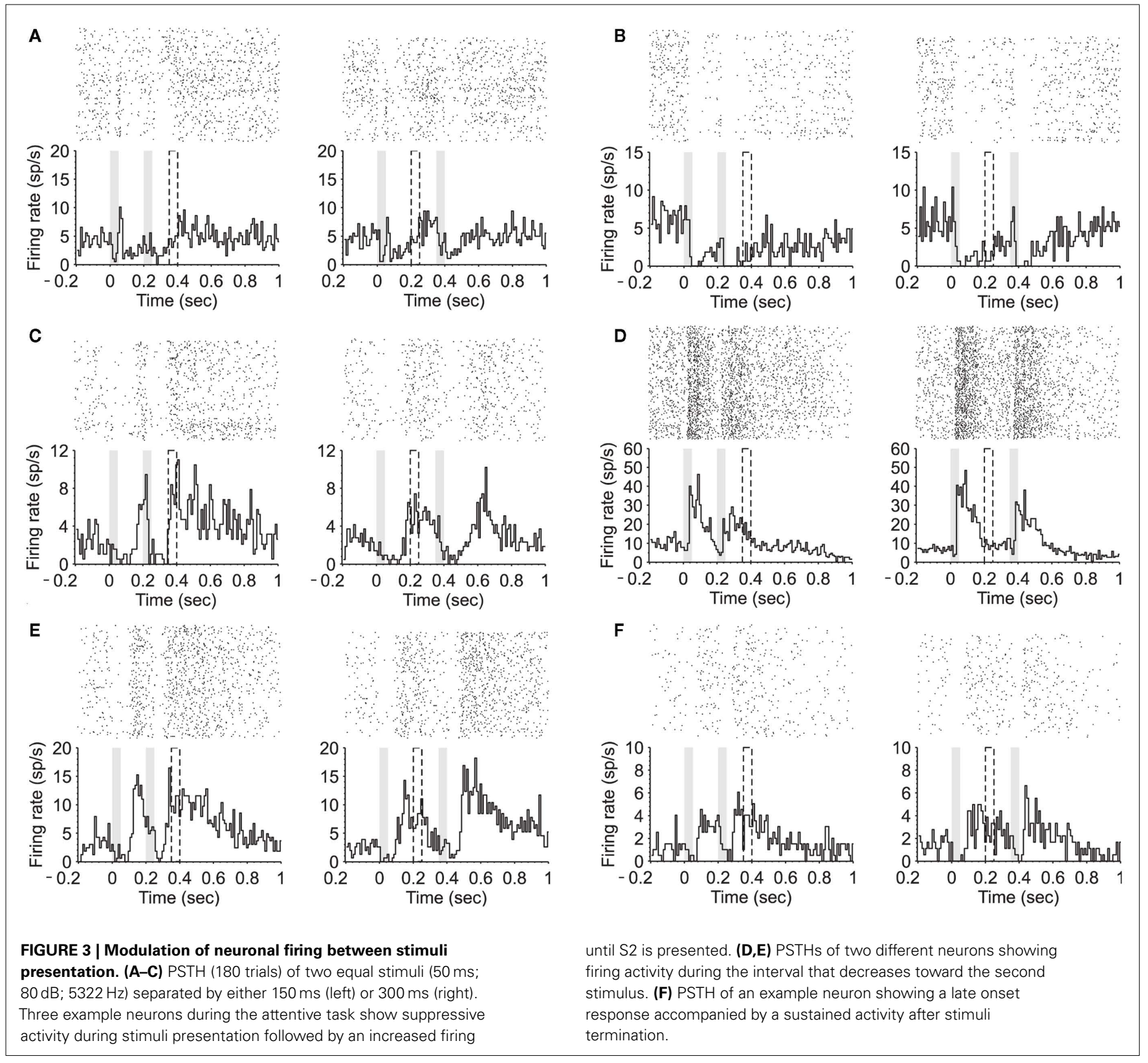

spontaneous discharge preceding the auditory stimulation, and no response to the auditory stimulus. However, a very large postdischarge followed each auditory stimulus. This unusual pattern of response took place during non-attentional trials. During attentional trials, those prominent post-discharges disappeared, and instead, the discharge preceding the first stimulus was increased, as did the example in Figure 4A. An enhanced firing rate preceding the occurrence of the first stimulus could be related to stimulus expectancy (Jaramillo and Zador, 2011) or to prediction of reward (Shuler and Bear, 2006), both described in early sensory cortices.

\section{DISCUSSION}

We recorded from neurons from the rat primary auditory cortex while the animal was performing an interval-discrimination

task. Here we report about 14 particular neurons that showed prominent responses during the intervals between stimuli, with firing rates that either increased or decreased toward the second stimulus. These neuronal discharges could be refered to as spontaneous activity, since they occurred while there was no auditory stimulation. However the term "spontaneous activity" has been avoided since these neuronal discharges were often associated to the preceding auditory responses, even if with a slow time course of over $150 \mathrm{~ms}$. The neuronal discharges occurring in the absence of auditory stimulation were enhanced by attention in 12 (out of 14) cases, while they were decreased in the remaining two.

Most of the studies of the effects of attention on auditory responses have focused on how phasic responses modulate their 

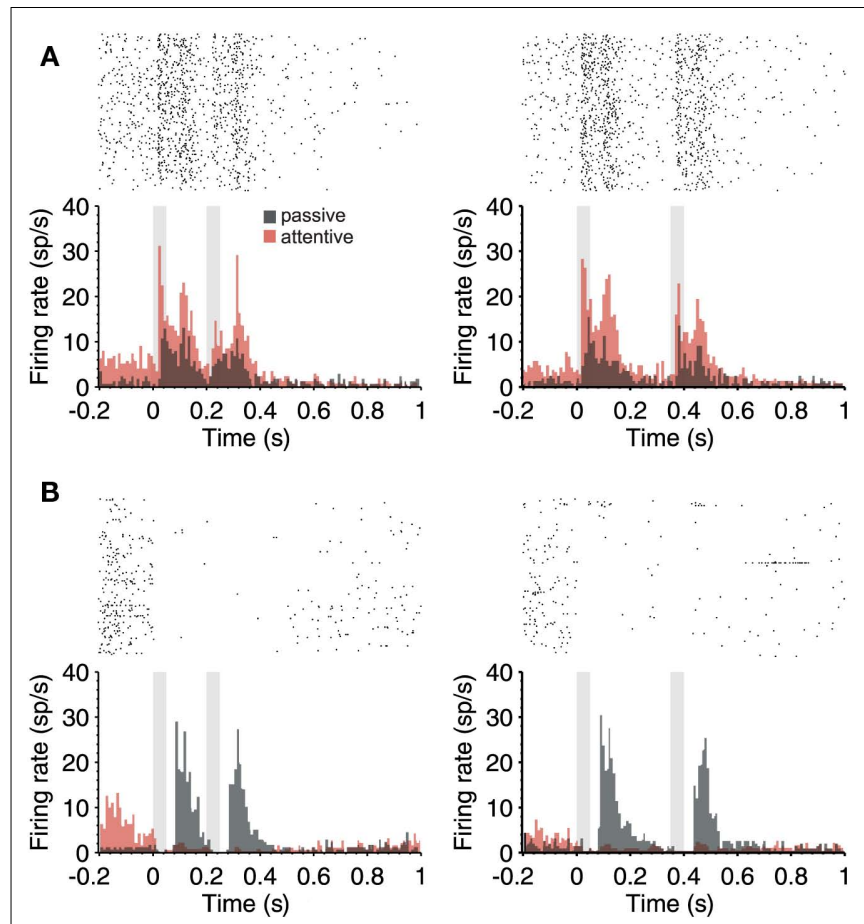

FIGURE 4 | Attention-modulated firing rate in the periods in between auditory stimuli. (A) PSTH (180 trials) of a single neuron during the passive (gray) and attentive (red) task where two identical stimuli $(50 \mathrm{~ms}$; $80 \mathrm{~dB} ; 5322 \mathrm{~Hz}$ ) separated by $150 \mathrm{~ms}$ (left) and $300 \mathrm{~ms}$ (right) were delivered. Notice that not only the response but also prominent responses in the absence of stimuli (preceding and between stimuli) are up regulated by attention. (B) Example neuron that had no auditory response, but a prominent and slow response after the termination of auditory stimuli during passive trials. This response was silenced during the attentive trials. However, notice that the activity preceding the first auditory stimulus was increased during attention (in red). In both cases (A,B), the raster plots correspond to the attentive trials. In all cases, 150 and $300 \mathrm{~ms}$ intervals were given randomly during the same sessions.

response properties according to the brain state (Hocherman et al., 1976; Pfingst et al., 1977; Benson and Hienz, 1978; Fritz et al., 2003; Schnupp et al., 2006; Yin et al., 2008; Otazu et al., 2009; Liu et al., 2010). Slow modulation of firing rate in the auditory cortex of the behaving monkey has been previously found to be related to the processing of stimuli, motor decision, or even reward (Selezneva et al., 2006; Yin et al., 2008; Brosch et al., 2011b), as it has been in primary visual cortex (Shuler and Bear, 2006). During behavioral experiments, this slow or sustained (up to several seconds) part of the response is related to event sequences during a task and provides a neuronal mechanism for anticipation and association of events related to hearing and relevant to behavior (Brosch et al., 2011b). Altogether, slow modulation of firing could complement the representation of the timing of auditory stimuli as well as the codification of stimuli by means of phasic responses. A similar pattern was reported by (Gottlieb et al., 1989; Durif et al., 2003; Yin et al., 2008).

Some studies have found no changes in spontaneous activity under attentional demands (Pfingst et al., 1977; Benson and
Hienz, 1978; Otazu et al., 2009). On the other hand, an increase in spontaneous firing rate at the end of the trial under attention with respect to the passive state has been reported, enhancement that could be reflecting motor-related aspects (Scott et al., 2007). Single units from auditory cortex have also been shown to have enhanced sustained responses preceding a target stimulus (Sakurai, 1990). Here we have shown that the spontaneous discharge is increased by attention in the period preceding the first stimulus in two neurons (Figures 4A,B).

The mechanisms for these slow modulations of firing rate are not known. One possibility would be that they reflect top-down modulation. Not only cortical, but also subcortical areas present modulation of spontaneous activity within tasks. Late trial neuronal activity in the monkey inferior colliculus has been described to be modulated by context, like a "reward expectation" signal (Metzger et al., 2006). Reward-modulation of the late activity after the end of the auditory stimulus has also been described in the rat auditory thalamus (Komura et al., 2005). A difference of these ramping activities with respect to the ones we have illustrated (Figure 2) is that the ramping-up here was preceding the second stimulus, and not the reward (Yin et al., 2008; Brosch et al., 2011a). The reward in our protocols occurred after the second stimulus, whenever the animal poked his nose in the correct side and thus triggered its delivery. It did not occur at a fixed time (usually after $1 \mathrm{~s}$ in the illustrated PSTHs). The ramping activity illustrated in Figure 2B between 0.6 and $1 \mathrm{~s}$ could be interpreted as such or associated to motor activity. We can speculate that the ramping-up activity in between stimuli (Figures 2A,B) could be rather associated to stimulus expectation or to intervalcomputation. In this respect, a recent study (Jaramillo and Zador, 2011) showed that neurons from the primary auditory cortex increased their firing rate as the target approached. This firing rate modulation reflected a temporal expectation which improved sound processing, therefore increasing the probability of obtaining a reward.

In all, neuronal firing in early auditory cortex in the absence of auditory stimulation could provide a neuronal mechanism for anticipation and memory, reflecting a learning process where consecutive sensory and behavioral events are associated with reinforcement. The slow modulation of ongoing firing during the interval between stimuli and the post-stimulus period could act as a mechanism to track and integrate time between stimuli presentations and be part of the neuronal basis of interval-categorization by means of tonic firing, particularly in attentive stages.

\section{ACKNOWLEDGMENTS}

We thank A. Zador, S. Jaramillo, G. Otazu for their help, and suggestions about the behavioral paradigm, to S. Aliagas for the animal training. We also thank L. Alonso, L. PérezMéndez, and D. Perez-Marcos for their programming work. Supported by a grant from the Ministerio de Ciencia e Innovación (BFU2008-01371/BFI) to MVS-V. MM-G and GD were supported by the European Union grant BRAINSCALES, by the Spanish Research Project SAF2010-16085 and by the CONSOLIDERINGENIO 2010 Programme CSD2007-00012, and EU FP7/20072013 under grant agreement 214728-2. 


\section{REFERENCES}

Abolafia, J. M., Vergara, R., Arnold, M. M., Reig, R., and Sanchez-Vives, M. V. (2011). Cortical auditory adaptation in the awake rat and the role of potassium currents. Cereb. Cortex 21, 977-990.

Benson, D. A., and Hienz, R. D. (1978). Single-unit activity in the auditory cortex of monkeys selectively attending left vs. right ear stimuli. Brain Res. 159, 307-320.

Brosch, M., Selezneva, E., and Scheich, H. (2011a). Representation of reward feedback in primate auditory cortex. Front. Syst. Neurosci. 5:5. doi:10.3389/fnsys.2011.00005

Brosch, M., Selezneva, E., and Scheich, H. (2011b). Formation of associations in auditory cortex by slow changes of tonic firing. Hear. Res. 271, 66-73.

Doron, N. N., Ledoux, J. E., and Semple, M. N. (2002). Redefining the tonotopic core of rat auditory cortex: physiological evidence for a posterior field. J. Comp. Neurol. 453, 345-360.

Durif, C., Jouffrais, C., and Rouiller, E. M. (2003). Single-unit responses in the auditory cortex of monkeys performing a conditional acousticomotor task. Exp. Brain Res. 153, 614-627.

Fritz, J., Elhilali, M., and Shamma, S. (2005). Active listening: taskdependent plasticity of spectrotemporal receptive fields in primary auditory cortex. Hear. Res. 206, 159-176.

Fritz, J., Shamma, S., Elhilali, M., and Klein, D. (2003). Rapid task-related plasticity of spectrotemporal receptive fields in primary auditory cortex. Nat. Neurosci. 6, 1216-1223.

Fritz, J. B., Elhilali, M., David, S. V., and Shamma, S. A. (2007). Does attention play a role in dynamic receptive field adaptation to changing acoustic salience in A1? Hear. Res. 229, 186-203.

Gottlieb, Y., Vaadia, E., and Abeles, M. (1989). Single unit activity in the auditory cortex of a monkey performing a short term memory task. Exp. Brain Res. 74, 139-148.

Hocherman, S., Benson, D. A., Goldstein, M. H. Jr., Heffner, H. E., and
Hienz, R. D. (1976). Evoked unit activity in auditory cortex of monkeys performing a selective attention task. Brain Res. 117, 51-68.

Hromadka, T., Deweese, M. R., and Zador, A. M. (2008). Sparse representation of sounds in the unanesthetized auditory cortex. PLoS Biol. 6, el6. doi:10.1371/journal.pbio.0060016

Hubel, D. H., Henson, C. O., Rupert, A., and Galambos, R. (1959). Attention units in the auditory cortex. Science 129, 1279-1280.

Imaizumi, K., Priebe, N. J., Sharpee, T. O., Cheung, S. W., and Schreiner, C. E. (2010). Encoding of temporal information by timing, rate, and place in cat auditory cortex. PLoS ONE 5, e11531. doi:10.1371/journal.pone.0011531

Jaramillo, S., and Zador, A. M. (2011). The auditory cortex mediates the perceptual effects of acoustic temporal expectation. Nat. Neurosci. 14, 246-251.

Kayser, C., Logothetis, N. K., and Panzeri, S. (2010). Millisecond encoding precision of auditory cortex neurons. Proc. Natl. Acad. Sci. U.S.A. 107, 16976-16981.

Kayser, C., Montemurro, M. A., Logothetis, N. K., and Panzeri, S. (2009). Spike-phase coding boosts and stabilizes information carried by spatial and temporal spike patterns. Neuron 61, 597-608.

Kayser, C., Petkov, C. I., and Logothetis, N. K. (2008). Visual modulation of neurons in auditory cortex. Cereb. Cortex 18, 1560-1574.

Komura, Y., Tamura, R., Uwano, T., Nishijo, H., and Ono, T. (2005). Auditory thalamus integrates visual inputs into behavioral gains. Nat. Neurosci. 8, 1203-1209.

Koontz, W. L. G., and Fukunaga, K. (1972). Asymptotic analysis of a nonparametric clustering technique. IEEE Trans. Comput. 21, 967-974.

Lemus, L., Hernandez, A., Luna, R., Zainos, A., and Romo, R. (2010). Do sensory cortices process more than one sensory modality during perceptual judgments? Neuron 67, 335-348.

Lemus, L., Hernandez, A., and Romo, R. (2009). Neural encoding of auditory discrimination in ventral premotor cortex. Proc. Natl. Acad. Sci. U.S.A. 106, 14640-14645.

Liu, Y., Qin, L., Zhang, X., Dong, C., and Sato, Y. (2010). Neural correlates of auditory temporal-interval discrimination in cats. Behav. Brain Res. 215, 28-38.

Lu, T., and Wang, X. (2004). Information content of auditory cortical responses to time-varying acoustic stimuli. J. Neurophysiol. 91, 301-313.

Metzger, R. R., Greene, N. T., Porter, K. K., and Groh, J. M. (2006). Effects of reward and behavioral context on neural activity in the primate inferior colliculus. J. Neurosci. 26, 7468-7476.

Nelken, I., Chechik, G., Mrsic-Flogel, T. D., King, A. J., and Schnupp, J. W. (2005). Encoding stimulus information by spike numbers and mean response time in primary auditory cortex. J. Comput. Neurosci. 19, 199-221.

Otazu, G. H., Tai, L. H., Yang, Y., and Zador, A. M. (2009). Engaging in an auditory task suppresses responses in auditory cortex. Nat. Neurosci. 12, 646-654.

Pfingst, B. E., O'Connor, T. A., and Miller, J. M. (1977). Response plasticity of neurons in auditory cortex of the rhesus monkey. Exp. Brain Res. 29, 393-404.

Polley, D. B., Heiser, M. A., Blake, D. T., Schreiner, C. E., and Merzenich, M. M. (2004). Associative learning shapes the neural code for stimulus magnitude in primary auditory cortex. Proc. Natl. Acad. Sci. U.S.A. 101, 16351-16356.

Recanzone, G. H. (2000). Response profiles of auditory cortical neurons to tones and noise in behaving macaque monkeys. Hear. Res. 150, 104-118.

Romanski, L. M., and Goldman-Rakic, P. S. (2002). An auditory domain in primate prefrontal cortex. Nat. Neurosci. 5, 15-16.

Sakurai, Y. (1990). Cells in the rat auditory system have sensory-delay correlates during the performance of an auditory working memory task. Behav. Neurosci. 104, 856-868.
Schnupp, J. W., Hall, T. M., Kokelaar, R. F., and Ahmed, B. (2006). Plasticity of temporal pattern codes for vocalization stimuli in primary auditory cortex. J. Neurosci. 26, 4785-4795.

Scott, B. H., Malone, B. J., and Semple, M. N. (2007). Effect of behavioral context on representation of a spatial cue in core auditory cortex of awake macaques. J. Neurosci. 27, 6489-6499.

Selezneva, E., Scheich, H., and Brosch, M. (2006). Dual time scales for categorical decision making in auditory cortex. Curr. Biol. 16, 2428-2433.

Shuler, M. G., and Bear, M. F. (2006). Reward timing in the primary visual cortex. Science 311, 1606-1609.

Weinberger, N. M. (2004). Specific longterm memory traces in primary auditory cortex. Nat. Rev. Neurosci. 5, 279-290.

Yin, P., Mishkin, M., Sutter, M., and Fritz, J. B. (2008). Early stages of melody processing: stimulussequence and task-dependent neuronal activity in monkey auditory cortical fields Al and R. J. Neurophysiol. 100, 3009-3029.

Conflict of Interest Statement: The authors declare that the research was conducted in the absence of any commercial or financial relationships that could be construed as a potential conflict of interest.

Received: 07 July 2011; accepted: 18 September 2011; published online: 19 October 2011.

Citation: Abolafia JM, Martinez-Garcia $M$, Deco $G$ and Sanchez-Vives $M V$ (2011) Slow modulation of ongoing discharge in the auditory cortex during an interval-discrimination task. Front. Integr. Neurosci. 5:60. doi: 10.3389/fnint.2011.00060

Copyright (c) 2011 Abolafia, MartinezGarcia, Deco and Sanchez-Vives. This is an open-access article subject to a nonexclusive license between the authors and Frontiers Media SA, which permits use, distribution and reproduction in other forums, provided the original authors and source are credited and other Frontiers conditions are complied with. 